\title{
Unexplained Diffuse Arteriosclerosis in a 26-Year-Old Patient
}

Jihed Anoun, Fatma Ben Fredj Ismai ${ }^{{ }^{*}}$, Anis Mzabi, Syrine Daadaa, Imene Ben Hassine, Monia Karmani, Hassen Hosni Mhiri, Amel Rezgui, Chedia Laouani Kechrid

Department of Internal Medicine, Sahloul Hospital, Faculty of Medicine, Sousse, Tunisia

*Corresponding author: Fatma Ben Fredj Ismail, Department of Internal Medicine, Sahloul Hospital, Faculty of Medicine, Sousse, Tunisia. Tel: +216 98504387; E-Mail: bfi.fatma@yahoo.fr

Citation: AnounJ, Ismail FBF, Mzabi A, Daadaa S, Hassine IB, et al. (2017) Unexplained Diffuse Arteriosclerosis in a 26-Year-Old Patient. Cardiolog Res Cardiovasc Med 2: 114. DOI: 10.29011/2575-7083.000014

Received Date: 15 April, 2017; Accepted Date: 15 May, 2017; Published Date: 25 May, 2017

\begin{abstract}
Arteriosclerosis is an alteration of the blood vessels whose walls calcify, lose their elasticity and thicken. The result is a decrease in circulating blood flow and ischemic manifestations. Calcification of arteries is a physiological phenomenon in the elderly but young subjects may also be affected. Indeed, some risk factors can cause arteriosclerosis. We report the case of a 26-year-old female patient with diffuse arteriosclerosis discovered accidentally, and whose exhaustive etiological investigation remained negative.
\end{abstract}

Keywords : Arteriosclerosis; Diffuse; Unexplained; Young

\section{Introduction}

Arteriosclerosis is an alteration of the blood vessels whose walls calcify, lose their elasticity and thicken. The result is a decrease in circulating blood flow and ischemic manifestations. Calcification of arteries is a physiological phenomenon in the elderly but young subjects may also be affected. Indeed, some risk factors can cause arteriosclerosis. These include classic cardiovascular risk factors, as well as systemic inflammatory diseases, connective tissue diseases, chronic hypocalcaemia. We report the case of a 26-year-old female patient with diffuse arteriosclerosis discovered accidentally, and whose exhaustive etiological investigation remained negative.

\section{Case Report}

BAO is 26 years old, single, student, non-smoking and active. She had neither significant family medical nor surgical history. She was followed since age 13 for rheumatoid arthritis without cardiac involvement, which was manifested by arthritis treated with non-steroidal anti-inflammatory drugs once or twice a year and once or both short corticosteroid treatment.

Two months prior to hospitalization, the patient had a right knee arthritis resistant to anti-inflammatory drugs. Radiography of the right knee revealed a calcification of the right popliteal artery (Figure 1,2,3). Arterial Doppler ultrasound of the right lower limb showed an irregular intimal calcification with diffuse medioclastic aspect of the femoro-popliteal and tibial arterial axis with significant hemodynamic repercussion and multiple loco regional substitution collaterals. There was, moreover, an aspect of calcifying tendonitis of the internal surface of the knee at the expense of the internal lateral ligament.

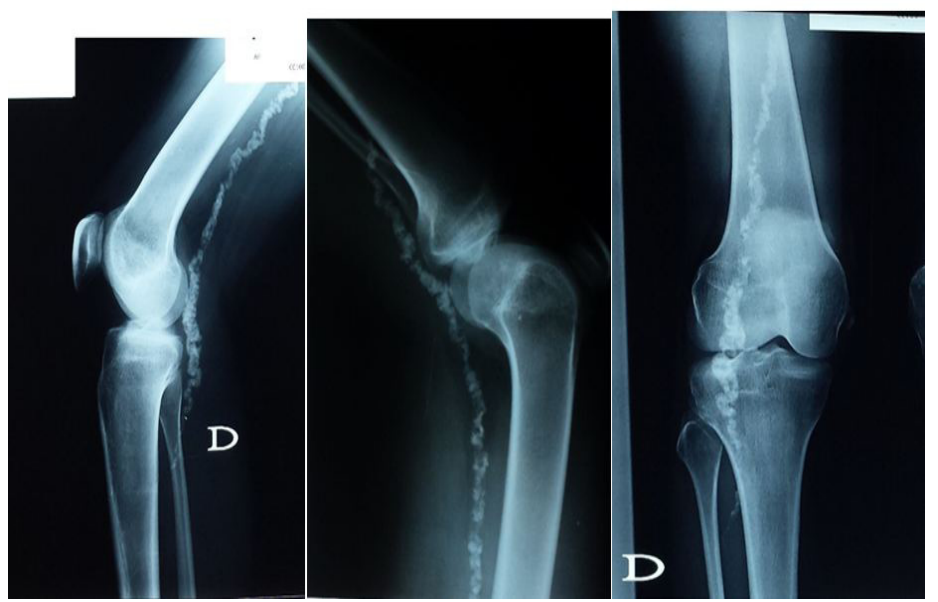

Figure 1,2\&3: Radiography of the Right Knee: Calcification of the Right Popliteal Artery.

She consulted in our department and the patient's interrogation revealed the presence of a phenomenon of Raynaud in its anoxic phase for several years and eliminated the use of drugs. Physical examination showed a weight of $45 \mathrm{~kg}$ with a body mass index of $18.9 \mathrm{~kg} / \mathrm{m} 2$ and a waist circumference of $72 \mathrm{~cm}$. The femoral and popliteal pulses were diminished on both sides and 


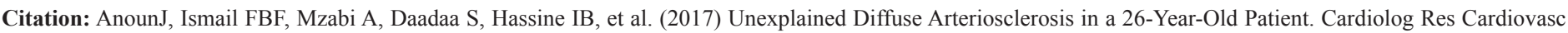
Med 2: 114. DOI: 10.29011/2575-7083.000014

the foot pulse was abolished on both sides. A coldness of both feet without cyanosis or necrosis was also noted. The rest of the exam was without anomalies.

Phosphate, calcium in the blood and urine, creatin, fasting blood, glucose, HbA1c, lipids, alkaline phosphatases, vitamin D, uricemia, homocysteinemia and a blood count were normal. Inflammatory biology (PEP and CRP) and immunoassay (ANAc, Rheumatoid Factor, and Anti-CCP Ac) were normal. A capillaroscopy revealed a capillary rarefaction without signs of chronic microangiopathy.

We carried out a radiological assessment which included the following examinations:

- Cervical ultrasound : Two parietal calcifications of the right thyroid lobe without lesion of the parathyroid.

- Upperlimbsarterial doppler ultrasonography : Absence of parietal calcifications.

- Angio-scan of the lowerlimbs : Extensive calcifications of the superficial femoralarteries and popliteal occludes along their paths, with deep femoralcollateral network draining a sural network of preserved quality (Figure 4,5,6).
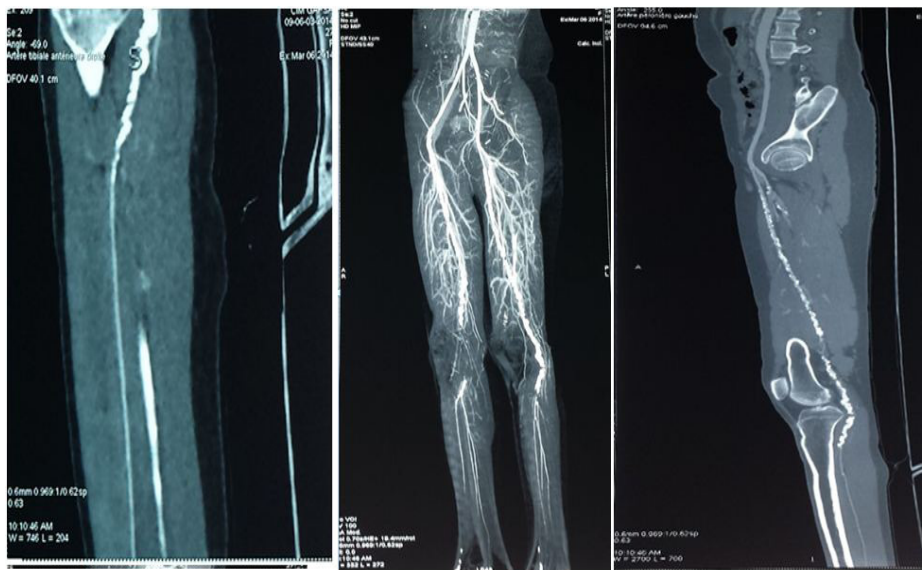

Figure 4,5\&6: Lower lumb Angio-CT: Extensive Calcifications of the Superficial Femoral Arteries and Occluded Poplites Along Their Paths, with a Network of Deep Femoral Collateral Draining a Sural Network of Preserved Quality.

- Angio-CT of the descending and ascending aorta: Multiple mediastinal and cervical calcifications confluent in places. Partial stenosing calcification of the left external carotid artery reducing light to $40 \%$. Non-obstructive parietal calcification of the right renalartery and the left internaliliac, reducing light by $50 \%$ and $30 \%$, respectively (Figure 7 ).

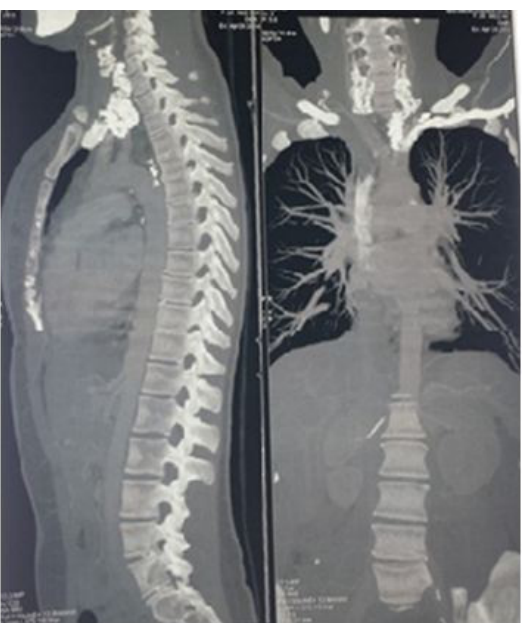

Figure 7: Angio-CT of the Descending and Ascending Aorta: Multiple Mediastinal and Cervical Calcifications Confluent in Places. Partial Stenosing Calcification of the Left External Carotid Artery Which Reduces Light To 40\%. Non-Obstructive Parietal Calcification of the right renal artery and the Left Internal Iliac, Reducing Light By 50 And 30\%, respectively.

- Brain CT scan : Bilateral punctiform micro calcifications of the lenticular nuclei.

- Cardiac ultrasound: without abnormalities.

A cutaneous biopsy showed discrete non-specific chronic inflammatory changes without histological signs in favor of a collagen or elastic connective tissue disorder. The patient was treated by statin, antiaggregant platelet and an anti-thrombotic. After 2-years of follow-up, its evolution is stationary; it has any new complaints or ischemic signs.

\section{Discussion}

It is a table of diffuse arteriosclerosis occurring in a girl of 26 years, having no significant cardiovascular risk factor, nor hypercalcemia. Few plausible etiologies explain this type of attack in the literature. We found no clinical or para-clinical evidence for inflammatory vascular disease: no inflammatory biological syndrome, no systemic manifestations other than the Raynaud phenomenon without real parietal vascular thickening in imaging. Nevertheless, the extensive and extra vascular distribution of calcifications argues against this etiology.

Calciphylaxis, or calcifying arterial disease, is a disease characterized by calcification followed by thrombosis of the small vessels of the skin and fat leading to necrotic ulcerations. It occurs in a particular area (chronic terminal renal failure in the hemodi- 


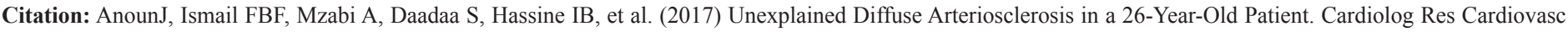
Med 2: 114. DOI: 10.29011/2575-7083.000014

alysis stage, hepatocellular insufficiency, alcoholic hepatic cirrhosis, primary hyperparathyroidism, regressive renal insufficiency, paraneoplastic). Unlike mediacalcosis, it is localized at the level of the intima of the arterioles. Initially considered a localized disease, it is increasingly considered a systemic disease [1,2]. But in our case, there was no predisposing factor for developing calciphylaxis, and the occurrence of large arteries and the normality of the cutaneous biopsy were against this diagnosis.

The inherited diseases of collagen may also be the cause of diffuse arterial calcifications. The diseases of Marfan and EhlersDanlos are rarely incriminated. On the other hand, Pseudo Xanthoma Elastica (PXE) has frequently been incriminated in the occurrence of diffuse arterial calcifications. It is a rare disease, due to a mutation on the ABCC6 gene (located on the short arm of chromosome 16) of autosomal recessive transmission. PXE is responsible for a calcifying juvenile arteriopathy that affects the proximal and distal axes with the development of collateral circulation. Patients are often pauci-symptomatic and ischemic complications are rare. A microangiopathic attack, objectified by capillaroscopy, is also present in this disease [3-8]. In our patient, although the type of vascular and extra vascular disease and the evolutionary mode were suggestive of PXE in the absence of obvious cardiovascular risk factors, she had no skin or ophthalmic signs of the disease, the cutaneous biopsy showed no alteration of the elastic fibers or calcium deposits, the family survey was negative and the genetic study in search of an abnormality of the ABCC6 gene is not accessible in Tunisia.

Another diagnosis was mentioned: the idiopathic calcified arteriopathy of the child. This is a rare condition seen in children under 3 years of age. It associates arterial calcifications accompanied by thromboses and occlusive intimal proliferation of coronary arteries with possible involvement of other vessels or the aorta. The clinical picture is non-specific, biologically, calcium and phosphorus are normal and the radiological assessment shows classically vascular calcifications. The prognosis is bleak with early mortality [9]. This diagnosis was not plausible in our patient before the age and the evolutionary mode of its disease. However, rare cases have been described in young adults [10]. The corticosteroid prescribed for its ancient arthritis was it behind the acceleration of arteriosclerosis? The literature is not in favor, such cases were not reported.

\section{Conclusion}

We present a case of calcifying arteriopathy in a 26-yearold girl whose diagnosis was accidentally discovered and whose exhaustive etiological investigation was negative.

\section{What is already know on this topic?}

- Arteriosclerosis in this age is exceptional.

- $\quad$ Predisposing factors of arteriosclerosis are absent in our case report.

- We didn't find an etiology to explain this arteriosclerosis in 26 years old.

\section{What this study adds?}

- Describe asymptomatic arteriosclerosis and its paraclinical founds.

- Discus causes of arteriosclerosis of young people.

\section{Authors' Contributions}

All authors contributed in conception, analysis, drafting the article and revising it critically for important intellectual content; and final approval of the version to be published.

\section{References}

1. Duval A, Moranne O, Vanhille P, Hachulla E, Delaporte E (2006) Calcium arteriolopathy (Calciphylaxis). Rev Med Int 27: 18495.

2. Agaajani S, Becker D, Loute G (2004) Lésions de calciphylaxie chez une patiente en insuffisance rénale terminale. Louvain Méd123: 210 216.

3. C Francès Maladies héréditaires du collagène et du tissu élastique. In: Traité de Médecine. 2004 éd.

4. Sene D (2016) Conduct in front of an arteriopathy of youngsubject, Hospital Boulevard, SFA: 47-83.

5. Perdu J, Champion K, Emmerich J, Fiessinger J-N (2004)Microangiopathy of elasticpseudoxanthoma. The Med press33: 518521.

6. Finger RP, Issa PC, Ladewig MS, Götting C, Szliska C, et al (2009) Pseudoxanthoma Elasticum: Genetics, Clinical Manifestations and TherapeuticApproaches. SurvOphthalmol54: 272285.

7. Nitschke Y, Rutsch F (2012) Generalizedarterial calcification of infancy and pseudoxanthomaelasticum: twosides of the same coin. Front Genet 3: 302.

8. Leftheriotis G, Kauffenstein G, Hamel JF, Abraham P, Le Saux O, et al. (2014) The contribution of arterial calcification to peripheral arterial disease in pseudoxanthomaelasticum. PloS One 9: e96003.

9. Michels (2016) Calcified Arteriopathy of the Idiopathic Child. Anabible.

10. Ferreira C, Ziegler S, Gahl W (2014) GeneralizedArterial Calcification of Infancy. 